\title{
Surface Characterization of the Hydroxy-Terminated Poly( $\epsilon$-caprolactone)/Poly(dimethylsiloxane) Triblock Copolymers by Electron Spectroscopy for Chemical Analysis and Contact Angle Measurements
}

\author{
H. Yıldırım Erbil, *,† Banu Yaşar, ${ }^{\ddagger}$ Şefik Süzer, ${ }^{\S}$ and Bahattin M. Baysal ${ }^{\dagger, \ddagger}$ \\ Marmara Research Center, Department of Chemistry, TÜBITAK, P.O. Box 21, \\ 41470 Gebze, Turkey, Department of Chemical Engineering, Boğaziçi University, \\ 80815 Bebek-İstanbul, Turkey, and Department of Chemistry, Bilkent University, \\ 06533 Bilkent-Ankara, Turkey
}

Received November 29, 1996. In Final Form: J une 13, $1997^{\otimes}$

\begin{abstract}
The surface composition and free energy properties of two grades of amphiphilic and semicrystalline triblock copolymers consisting of a poly(dimethylsiloxane) (PDMS) midblock ( $\mathrm{M}_{\mathrm{w}} \sim 2300$ ) coupled to poly( $\epsilon$-caprolactone) $(\mathrm{PCL})$ end blocks having differing molecular weights $\left(\mathrm{M}_{\mathrm{w}} \sim 2000\right.$, sample $\mathrm{P} 3$, and $\mathrm{M}_{\mathrm{w}} \sim$ 3000, sample P2) and homopolymer PCL $\left(\mathrm{M}_{\mathrm{w}} \sim 40000\right)$ were investigated by Fourier transform infrared, spectroscopy, el ectron spectroscopy for chemical analysis (ESCA), and contact angle measurements using critical surface tension, one-liquid and two-liquid methods. ESCA showed that the molar concentration of PDMS increased from $36.5 \%$ in the bulk up to $70.2 \%$ in the surface for sample P2 and from $46.3 \%$ in the bulk up to $79.2 \%$ in the surface for sample P3 in high vacuum. This indicates that the lower surface energy PDMS microdomains were segregated in the surface region to minimize the surface energy of the copolymer. The longer the PCL block, the higher the phase separation. One-liquid contact angle results were evaluated by using van Oss, Good, and Chaudhury's Lifshitz-van der Waals and Lewis acid-base (AB) methodology, and it was determined that the basicity surfacetensi on coefficients $\left(\gamma_{\mathrm{s}}{ }^{-}\right)$of thecopolymers decreased with the increase of the PDMS content at the surface, a result in agreement with the ESCA results but not proportional to them, indicating that the surfaces of the copolymers are highly mobile and molecular rearrangement takes place upon contacting with a polar testing liquid drop. The strong $A B$ interaction between the basic carboxyl groups of PCL segments with the Lewis acidic groups of the polar liquids restructured the surface molecular composition at thecontact area by increasing PCL and decreasing PDMS concentration in polar environments. The two-liquid contact angle method was also applied, and it was determined that $\gamma_{s}{ }^{-}$decreased inverse proportionally with the increase of PDMS segments. Also, it was realized that the molecular restructuring did not take place in the two-liquid method.
\end{abstract}

\section{Introduction}

Poly(dimethylsiloxanes) (PDMS) arematerials of broad applicability in a variety of industrial areas because of their well-known surface modifying properties. ${ }^{1-3}$ They have extremely low glass transition temperatures $(-120$ ${ }^{\circ} \mathrm{C}$ ), very high chain flexibility, good oxidative, thermal, and UV stability, water repellancy, high gas permeability, biocompatibility (physiological inertness), and low surface tension. Dueto their very largemol ar volumes combined with the very low cohesiveenergy densities (intermol ecular interactions) and high chain flexi bilities, PDMS has a very low solubility parameter and low surface tension which is around $16-22 \mathrm{~mJ} / \mathrm{m}^{2}$, varyingaccording tothemolecular weight of PDMS. 3,4 This value is at least $10 \mathrm{~mJ} / \mathrm{m}^{2}$ lower than that of many other polymers. Despite their many unique properties, pure poly(dimethylsiloxanes) are mechanically too weak to be used directly as a polymer and they show cold flow even at very high molecular weights $\left(\mathrm{M}_{\mathrm{n}} \sim 500000\right)$.

In addition, while its surface properties render PDMS very attractive for modification of other surfaces and

* Corresponding author: fax, 0262 6412309; e-mail, hyerbil@ mam.gov.tr.

† TÜBITAK.

‡ Boğaziçi University.

$\S$ Bilkent University.

${ }^{\otimes}$ Abstract published in Advance ACS Abstracts, September 1, 1997.

(1) J avis, N. L. J . Phys. Chem. 1966, 70, 3027.

(2) Owens, M.J.; Kendrick, T. C. Macromolecules 1970, 3, 458.

(3) Yılgör, I.; McGrath, J. E. Adv. Polym. Sci. 1988, 86, 1.

(4) Yılgör, I.; Steckle, W. P.; Yılgör, E.; Freelin, R. G.; Riffle, J . S. J .

Polym. Sci., Polym. Chem. Ed. 1989, 27, 3673. interfaces, its low solubility parameter causeit to behighly immiscible (thermodynamic incompatibility) with most other polymers. In practice, PDMS has little use for surface modification by blending, since it tends to be rejected from the matrix. An effective way to increase the compatibility of such blends is to form copolymers of siloxanes with carbon-based polymers. ${ }^{4,5}$ These can be obtained through the reaction of organofunctionally terminated siloxane oligomers with various organic monomers, resulting in multiphase, block, segmented, or graft copolymers. ${ }^{4}$ These copolymers are useful additives for the surface modification of some bulk polymers.

It is well-known that the surface dynamics of polymer solids are consi derably different from those of more rigid materials such as metals and ceramics, due to the high mobility of macromolecules at the surface. Also, the composition of the copolymer surface is not necessarily the same as the composition in the bulk. Generally, copolymers and polymer blends havea surfacecomposition that differs greatly from their bulk composition since components of lower surface energy al ways tend to enrich at the surface to mini mize the free energy of the system. ${ }^{6}$ As a result of their incompatibility and surface activity when siloxane-containing copolymers are blended with various organic polymers, the air-polymer surfaces of the resulting systems are dominated by the low surface free energy siloxanes. It is a common observation that even at very low levels of bulk siloxane content $(0.5-5.0 \%$ by weight) theresultant blends display completely silicone-

(5) Lovinger, A. J .; Han, B. J .; Padden, F. J .; Mirau, P. A. J . Polym. Sci., Polym. Phys. Ed. 1993, 31, 115.

(6) Pertsin, A. J .; Gorelova, M. M.; Levin, V. Y.; Makarova, L. I. J . Appl. Polym. Sci. 1992, 45, 1195. 
like surface properties. ${ }^{2,7}$ The studies on the surface properties of the poly(dimethylsiloxane) block copolymers themselves as well as their mixtures with homopolymers have been carried out extensively by several groups. ${ }^{8-12}$ Most works revealed that the component with lower surface free energy compared with that of the other component was preferentially concentrated at the polymer-air interface.

On the other hand, the surfaces of polymeric solids can adopt different surface configurations in different environments soas to increasetheir interaction with thelatter and thereby minimize the total free energy of the system. ${ }^{13-16}$ By rotational and translational motions of chains and chain segments, polymers mi nimizethedensity of polar groups at the surface in a nonpolar environment such as air, ${ }^{17}$ whereas in an aqueous environment it is energetically more favorable for the polymer surface to become enriched in polar groups and reduce the density of nonpolar groups. ${ }^{18}$ In systems containing hydrophilic phases of submicroscopic dimensions such as common diblock and triblock copolymers, gi ven sufficient mobility, the hydrophilic phase will dominatetheinterface in polar medium, whereas the hydrophobic phase will dominate in air. ${ }^{16}$

The instrumental surface analysis techniques which have been developed over the last 30 years are being increasingly applied for thestudy of polymer surfaces and interfaces. ${ }^{5-12,15,16}$ These methods such as X-ray photoelectron spectroscopy (XPS) or the other name, electron spectroscopy for chemical analysis (ESCA), Auger el ectron spectroscopy (AES), and secondary i on mass spectroscopy (SIMS) require ultrahigh vacuum environments. The assumption is generally made that the polymer surface is indeed stable and that the results of the analysis are applicable to the non-vacuo environments wherein the polymer surface is usually applied. Such an assumption is often invalid. ${ }^{16}$ In order to examinethepolymer surface restructuring process in the application medium, the contact angles provide an attractive basis for a quantitative description of polymer surface dynamics since they al low the determination of surface properties at the very surface of solid polymers and they probethesurfacelayers toa depth comparabletotherange of theinterfacial forces involved in surface restructuring. The contact angles relate to the fractions of nonpolar and polar structural elements on a surface. ${ }^{19}$

In this study, wehavechosen hydroxy-terminated poly( $\epsilon$-caprolactone)/poly(dimethylsiloxane) triblock copolymers (PCL/PDMS/PCL) as the amphiphilic block copolymer (having both polar and nonpolar character) to be investigated. Thesynthesis, characterization, solid state

(7) Lelah, M. D.; Cooper, S. L. In Polyurethanes in Medicine; CRC Press, Inc.: Boca Raton, FL, 1986.

(8) Clark, D. T.; Peeling, J . J . Polym. Sci., Polym. Chem. Ed. 1976, 14, 543.

(9) Chen, X.; Gardella, J . A.; Ho, T.; Wynne, K. J . Macromolecules 1995, 28, 1635. 2206.

(10) Chen, X.; Gardella, J . A.; Cohen, R. E. Macromol ecules 1994, 27,

(11) Smith, S. D.; DeSimone, J . M.; Dwight, D. W.; McGrath, J . E. Macromol ecules 1992, 25, 2575 .

(12) Benrashid, R.; Nelson, G. L.; Linn, J . H.; Hanley, K. H.; Wade,

W. R. J . Appl. Polym. Sci. 1993, 49, 523.

(13) Ruckenstein, E.; Gourisankar, S. V. J . Colloid Interface Sci. 1985, 107, 488.

(14) Andrade, J . D., Ed. Polymer Surface Dynamics; Plenum Press: New York, 1988.

(15) Chatelier, R. C.; Xie, X.; Gengenbach, T. R.; Griesser, H. J . Langmuir 1995, 11, 2576.

(16) Andrade, J . D.; Chen, W. Y. Surf. Interface Anal. 1986, 8, 253.

(17) Baszkin, A.; Ter-Minassian-Saraga, L. Polymer 1974, 15, 759.

(18) Holly, F. J .; Refojo, M. J. Biomed. Mater. Res. 1975, 9, 315.

(19) J ohnson, R. E.; Dettre, R. H. In Surface and Colloid Science; Matijevic, E., Ed.; Wiley Intersience, New York, 1969; Vol. 2, p 85.
Table 1. Bulk Composition of Samples

\begin{tabular}{clrrrl}
\hline sample & description & $\begin{array}{r}\text { \% wt } \\
\text { PDMS }\end{array}$ & $\begin{array}{r}\text { \% mol } \\
\text { PDMS }\end{array}$ & $\begin{array}{c}\text { \% mol } \\
\text { PCL }\end{array}$ & $\begin{array}{c}\text { atomic } \\
\text { Si/C }\end{array}$ \\
\hline P1 & Pure PCL & & & 100.0 & 0 \\
P2 & H-Si 6460 & 27.0 & 36.5 & 63.5 & 0.08 \\
P3 & H-Si 6440 & 35.7 & 46.3 & 53.7 & 0.11 \\
P4 & Pure PDMS & 100.0 & 100.0 & & 0.50
\end{tabular}

morphology, thermal properties, and phase behavior of this bl ock copolymers have only recently been reported. ${ }^{4,5}$ Although, there are some studies on the surface characterization of other poly(dimethylsiloxane) block copolymers, ${ }^{8-10,12}$ they are mostly based on instrumental techniques such as ESCA, F ourier transform infrared (FTIR), and scanning el ectron microscopy (SEM). Only onepaper, investigating poly(dimethylsiloxane)/polystyrene block copolymers reported the use of contact angles and also the critical surfacetension values calculated from contact angles. ${ }^{8}$ However, the information derived from liquid drop contact angl es on a surface was increased very much by the combination of Lifshitz-van der Waals and acidbase theories with the contact angle theory in recent years. ${ }^{20-25}$ In this study, the comparison of the results derived from instrumental techniques (ESCA and FTIR) with theresults obtained from contact angles is intended. For this purpose, homopolymer PCL and two grades of triblock PLC/PDMS/PLC copolymers having only different $\mathrm{PCL}$ molecular lengths were investigated.

\section{Experimental Section}

Materials. The block copolymers studied in this work are commercial triblock poly( $\epsilon$-caprolactone)-poly(dimethylsiloxane)-poly( $\epsilon$-caprolactone) copolymers, kindly supplied by $\mathrm{TH}$. Goldschmidt A.G., Essen, Germany, under the trade name of Tegomer having the following structural formula.

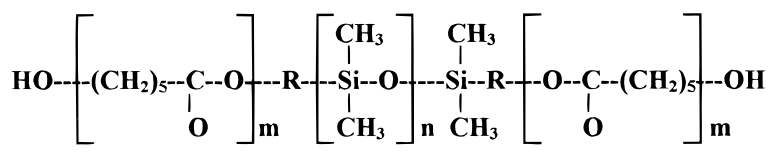

Twoentities of Tegomers wereexamined: $\mathrm{H}-\mathrm{Si}-6440, \mathrm{M}_{\mathrm{n}}=6500$ $\pm 600, \mathrm{n} / \mathrm{m}(\mathrm{calcd})=30 / 18, \mathrm{mp}=54 \pm 1^{\circ} \mathrm{C} ; \mathrm{H}-\mathrm{Si}-6460, \mathrm{M}_{\mathrm{n}}=8500$ $\pm 800, \mathrm{n} / \mathrm{m}(\mathrm{calcd})=30 / 27, \mathrm{mp}=57 \pm 1^{\circ} \mathrm{C}$. I n both of these block copolymers, the molecular weights of PDMS blocks was kept constant ( 2300), differing only the molecular weights of PCL blocks, which were $\sim 2000$ and $\sim 3000$, respectively. A sample of poly( $\epsilon$-caprolactone) obtained from Polysciences, I nc. (catalog no. 07039), having $M_{w}=35000-45000$ and poly(dimethylsiloxane) having $M_{w} \sim 10000$ areal so examined. The samples were designated as P1, P2, P3, and P4, and their bulk compositions are given in Table 1. All chemicals were reagent grade and used as received.

FTIR. FTIR spectra were recorded using a thin film of each sample prepared on $\mathrm{NaCl}$ disks by melting of appropriateweight. A BOMEM M 102 series instrument is used in absorbance mode with $4 \mathrm{~cm}^{-1}$ resolution and an average of 256 scans.

ESCA. Electron spectroscopy for chemical analysis or X-ray photoelectron spectroscopy (XPS) data were obtained with a KRATOS ES300E SCA instrument under ultrahigh vacuum, 10-8 Torr. Samplefilms wereprepared on theprobe of this instrument. Mg K $\alpha$ X-rays (1253.6 eV) were used to record the (XPS) and Auger spectra at two different takeoff angles $\left(90\right.$ and $\left.20^{\circ} \mathrm{C}\right)$. The

(20) Good, R. J . In Contact Angle, Wettability and Adhesion; Mittal, K. L., Ed.; VSP Press: Utrecht, 1993; p 3.

(21) Van Oss, C. J . Interfacial Forces in Aqueous Media; Marcel Dekker, Inc.: New York, 1994.

(22) Van Oss, C. J .; Chaudhury, M. K.; Good, R.J . Chem. Rev. 1988, 88, 927.

(23) Erbil, H. Y. Langmuir 1994 10, 286.

(24) Erbil, H. Y. Langmuir 1994 10, 2006.

(25) Erbil, H. Y. In Handbook of Surface and Colloid Chemistry; Birdi, K. S., Ed.; CRC Press, Inc.: Boca Raton, FL, 1997; Chapters 2 and 9. 
spectra of PCL and PDMS compare quite well with the highresolution reference XPS spectra of organic polymers compiled by Beamson and Briggs. ${ }^{26}$

Contact Angle Goniometry. Contact angl emeasurements were made by a KERNCO Model G-III contact angle meter at $25^{\circ} \mathrm{C}$ and ambient humidity. Only advancing angles were determined immediately. In the oneliquid method (air-liquid drop-polymer system), contact angles of water, glycerol, ethylene glycol, formamide, and paraffin oil drops were measured. In the two-liquid method (liquid-immiscible liquid drop-polymer system), two sets of measurements were carried out: water, formamide, ethylene glycol drops on the polymer in paraffin oil bulk phaseand paraffin oil drops in water, formamide, ethylene glycol bulk phases. All the reported contact angles were an average of three measurements and deviated within $\pm 2^{\circ}$.

All the polymers weresupplied in pellet form. Solvent casting was attempted with various solvents (and solvent mixtures), but smooth film surfaces could not be obtained. Then, smooth films were prepared by melting the pellets on carefully cleaned glass slides at $\sim 60^{\circ} \mathrm{C}$ and then by cooling. The glass sides of the samples wereused for measurements becauseair sides had some roughness.

\section{Contact Angle Theory}

The surface tension of polymers cannot be measured directly because of the elastic and viscous restraints of the bulk phase which necessities the use of indirect methods such as contact angle measurement. Several semiempirical approximations are currently available to evaluatethesurfacetension of polymers from contact angle data such as geometric-mean, harmonic-mean, and equation of state approaches. ${ }^{25}$ Recently, van Oss, Good, and Chaudhury devel oped a more advanced approach based on the Lifshitz theory ${ }^{27}$ of the attraction between macroscopic bodies to estimate the free energy of adhesion between two condensed phases. ${ }^{20-22}$ They suggested that a solid surface consists of two terms, one is Lifshitz-van der Waals (LW) interaction term, $\gamma \mathrm{LW}$, comprising dispersion, dipolar, and induction forces, and the other term is acid-base interaction term, $\gamma^{\mathrm{AB}}$, comprising all the electron donor-acceptor interactions and hydrogen-bonding. The two terms are additive to yield surface or interfacial tension term of both solids and liquids

$$
\gamma_{12}=\gamma_{12}{ }^{\mathrm{LW}}+\gamma_{12}^{\mathrm{AB}}
$$

Young's equation describes the thermodynamic equilibrium of the three surface free energies $\gamma_{S V}, \gamma_{S L}$, and $\gamma_{L V}$ existing at the phase boundaries of a drop of liquid at rest on a solid surface

$$
\gamma_{\mathrm{LV}} \cos \theta=\gamma_{\mathrm{SV}}-\gamma_{\mathrm{SL}}
$$

where $\gamma$ is the surface tensi on and the subscripts SV, SL, and LV refer tothesolid-vapor, solid-liquid, and liquidvapor interfaces, respectively. ${ }^{28}$

Dupréintroduced the concepts of work of cohesion, Wc, and work of adhesion, $W^{a}$, that the work required to separatetheunit surface of the samesubstance(cohesion) and different substances (adhesion). ${ }^{29}$ At present, the thermodynamic notation, in terms of free energy, G, is preferably employed instead of W in order to emphasize thefact that $\gamma$ is, physically, the free energy per unit area, as well as force per unit length and also to keep the signs of the physical quantities straight. ${ }^{20}$ Then it may be written

(26) Beamson, G.; Briggs, D. High Resolution XPS of Organic Polymers; Wiley: New York, 1993.

(27) Lifshitz, E. M. Sov. Phys. J ETP 1956, 2, 73

(28) Young, T. Philos. Trans. R. Soc. London 1805, 95, 65.

(29) Dupré, A. In TheorieM ecaniquedela Chaleur; Gauthier-Villar: Paris, 1869; p 368.

$$
\begin{gathered}
\Delta G_{11}{ }^{c}=-W^{c}=-2 \gamma_{1} \\
\Delta G_{12}{ }^{a}=-W^{a}=\gamma_{12}-\gamma_{1}-\gamma_{2}
\end{gathered}
$$

Equation 4 may be written as

$$
\Delta \mathrm{G}_{\mathrm{SL}}{ }^{\mathrm{a}}=\gamma_{\mathrm{SL}}-\gamma_{\mathrm{S}}-\gamma_{\mathrm{L}}
$$

Assuming that $\gamma_{\mathrm{L}}=\gamma_{\mathrm{LV}}$ and $\gamma_{\mathrm{S}}=\gamma_{\mathrm{SV}}$ and by combining eqs 2 and 5 , one obtains the Young-Dupré equation:

$$
\Delta \mathrm{G}_{\mathrm{SL}}{ }^{\mathrm{a}}=-\gamma_{\mathrm{LV}}(1+\cos \theta)
$$

When the van Oss-Good theory is applied to the Dupré equation, one obtains

$$
\Delta G_{S L}{ }^{a}=\left(\Delta G_{S L}{ }^{a}\right)^{L W}+\left(\Delta G_{S L}{ }^{a}\right)^{A B}
$$

The hypothesis was proposed that the LW component of adhesion is equal to the geometric mean of the LW components of cohesion of the separate phases

$$
\left(\Delta G_{S L}{ }^{a}\right)^{L W}=\left[\left(\Delta G_{S}{ }^{c}\right)^{L W}\left(\Delta G_{L}{ }^{c}\right)^{L W}\right]^{1 / 2}
$$

Since $\left.\left(\Delta \mathrm{G}_{\mathrm{S}}\right)^{\mathrm{C}}\right)^{\mathrm{LW}}=-2 \gamma_{\mathrm{S}}{ }^{\mathrm{LW}}$ and $\left(\Delta \mathrm{G}_{\mathrm{L}}{ }^{\mathrm{C}}{ }^{\mathrm{LW}}=-2 \gamma_{\mathrm{L}}{ }^{\mathrm{LW}}\right.$; eq 8 becomes

$$
\left(\Delta G_{S L}^{a}\right)^{L W}=-2\left(\gamma_{S}^{L W} \gamma_{L}^{L W}\right)^{1 / 2}
$$

By combining eqs 5 and 9 in the LW form, one obtains

$$
\gamma_{S L}{ }^{L W}=\gamma_{S}{ }^{L W}+\gamma_{L}{ }^{L W}-2\left(\gamma_{S}{ }^{L W} \gamma_{L}^{L W}\right)^{1 / 2}
$$

or

$$
\gamma_{S L}{ }^{L W}=\left\{\left(\gamma_{S}^{L W}\right)^{1 / 2}-\left(\gamma_{L}^{L W}\right)^{1 / 2}\right\}^{2}
$$

However, the acid-base interaction term (AB) cannot be combined with a geometric mean rule. In many liquidsolid interfaces, in addition to LW interactions, hydrogen bonding and other electron acceptor-electron donor interactions or Lewis acid-base interactions often occur. Unlike LW interactions, AB interactions are essentially asymmetrical and can only be satisfactorily treated by taking that asymmetry into account. ${ }^{20}$

Theelectron acceptor and el ectron donor parameters of the $\gamma_{i}{ }^{A B}$ are thus expressed as, respectively $\gamma_{i}{ }^{+}$and $\gamma_{i}{ }^{-}$so that

$$
\gamma_{i}^{A B}=2\left(\gamma_{i}^{+} \gamma_{i}^{-}\right)^{1 / 2}
$$

A molecule can be both a Lewis acid and a Lewis base as with water and termed as "bi polar". If either the acidic or basic property is negligible and the other property is appreciable, the substance is termed as "monopolar". As an example, chloroform is monopolar acidic and ether is monopolar basic. An "apolar" substance exhibits neither appreciable acidic nor basic properties, such as paraffin oil. If the interacting substances are monopolar in the same sense, e.g., both being acidic or basic, then there is noacid-baseinteraction across theinterface. If they are monopolar in the opposite sense, one being a Lewis acid and the other a Lewis base, or if one of the components is monopolar and the other is bipolar (such as water), then an acid-baseinteraction is present. Theseexamples explain the importance of the asymmetry in $A B$ interactions.

Thepostulated combining rulefor acid-baseinteraction across an interface is ${ }^{20}$ 


$$
\left(\Delta G_{S L}{ }^{a}\right)^{A B}=-2\left\{\left(\gamma_{S}^{+} \gamma_{L}^{-}\right)^{1 / 2}+\left(\gamma_{S}^{-} \gamma_{L}^{+}\right)^{1 / 2}\right\}
$$

and

$$
\begin{aligned}
\gamma_{\mathrm{SL}}{ }^{\mathrm{AB}}=2\left\{\left(\gamma_{\mathrm{S}}^{+} \gamma_{\mathrm{S}}{ }^{-}\right)^{1 / 2}+\right. & \left(\gamma_{\mathrm{L}}^{+} \gamma_{\mathrm{L}}^{-}\right)^{1 / 2}- \\
& \left.\left(\gamma_{\mathrm{S}}^{+} \gamma_{\mathrm{L}}^{-}\right)^{1 / 2}-\left(\gamma_{\mathrm{S}}{ }^{-} \gamma_{\mathrm{L}}^{+}\right)^{1 / 2}\right\}
\end{aligned}
$$

or

$\gamma_{\mathrm{SL}}{ }^{\mathrm{AB}}=2\left\{\left(\gamma_{\mathrm{S}}^{+}\right)^{1 / 2}-\left(\gamma_{\mathrm{L}}^{+}\right)^{1 / 2}\right\}\left\{\left(\gamma_{\mathrm{S}}{ }^{-}\right)^{1 / 2}-\left(\gamma_{\mathrm{L}}^{-}\right)^{1 / 2}\right\}$

This form of combining rulewas first suggested by Small; 30 it was also derived from molecular orbital theory. ${ }^{31}$ By combining eqs 3 and 11, one obtains

$$
\left(\Delta G_{i}\right)^{A B}=-4\left(\gamma_{i}^{+} \gamma_{i}^{-}\right)^{1 / 2}
$$

that may be applied both to solid and liquid cohesion.

One interesting consequence of eq 13 is that the contribution of $A B$ interaction to interfacial tension may be negative. 20,21

By combining eqs 7, 9, and 12 , oneobtains the compl ete combining rule that has been postulated for $L W$ and $A B$ components together

$$
\begin{aligned}
\Delta \mathrm{G}_{\mathrm{SL}}{ }^{\mathrm{a}}=-2\left\{\left(\gamma_{\mathrm{S}}{ }^{\mathrm{LW}} \gamma_{\mathrm{L}}{ }^{\mathrm{LW}}\right)^{1 / 2}+\left(\gamma_{\mathrm{S}}{ }^{+} \gamma_{\mathrm{L}}{ }^{-}\right)^{1 / 2}+\right. \\
\left.\left(\gamma_{\mathrm{S}}{ }^{-} \gamma_{\mathrm{L}}{ }^{+}\right)^{1 / 2}\right\}
\end{aligned}
$$

If we combine the Y oung-Dupré equation (eq 6) with eqs 7,9 , and 15 , weobtain the general contact angl eequation, for liquid $L$ on solid $S$

$$
\begin{array}{r}
\gamma_{L}(1+\cos \theta)=2\left\{\left(\gamma_{S}{ }^{L W} \gamma_{L}^{L W}\right)^{1 / 2}+\left(\gamma_{S}^{+} \gamma_{L}^{-}\right)^{1 / 2}+\right. \\
\left.\left(\gamma_{S}^{-} \gamma_{L^{+}}\right)^{1 / 2}\right\}
\end{array}
$$

The surface tension components of the polymeric solids, $\gamma_{\mathrm{s}}{ }^{\mathrm{LW}}, \gamma_{\mathrm{s}}{ }^{+}$, and $\gamma_{\mathrm{s}}{ }^{-}$can be calculated using at least three liquid drops on the solid and by simultaneous solution of eq 16. $\left(\gamma_{\mathrm{L}}{ }^{\mathrm{LW}}, \gamma_{\mathrm{L}}^{+}\right.$, and $\gamma_{\mathrm{L}}{ }^{-}$values for liquid drops are calculated in advance using $\gamma_{\mathrm{w}^{\mathrm{WW}}}=21.18$ and $\gamma_{\mathrm{w}}{ }^{+}=\gamma_{\mathrm{w}^{-}}$ $=25.5 \mathrm{~mJ} / \mathrm{m}^{2}$ values for water and by deriving the other liquid parameter values from water. ${ }^{20-22}$ )

Two-Liquid Method. For high-energy surfaces such as metals, metal oxides, silica, etc., the wetting with liquid is generally complete, and since the test liquids spread on them, the determination of surface tension from simple contact anglemeasurements becomes impossible. In order toprevent thespread of thewettingliquids, theair medium was replaced by a second immiscible liquid on the solid surface, such as a water drop in hydrocarbons. This method was also applied to low-energy polymer surfaces. ${ }^{24,25,32-34}$ When Young's equation is applied to the two-liquid method, one obtains

$$
\gamma_{\mathrm{SL}_{2}}=\gamma_{\mathrm{SL}_{1}}+\gamma_{\mathrm{L}_{1} \mathrm{~L}_{2}} \cos \theta
$$

where $L_{1}$ is the sessile liquid drop on a polymer surface, $S$, and $L_{1}$ liquid drop is immersed in the other immiscible liquid $L_{2}$, and $\gamma_{S_{2}}, \gamma_{S_{1}}$, and $\gamma_{L_{1} L_{2}}$ are, respectively, the interfacial tension of $S-L_{2}, S-L_{1}$, and $L_{1}-L_{2}$ interfaces.

When water and other polar immiscible liquid drops are formed on polymer surfaces in hydrocarbon (HC)

(30) Small, P. A. J . Appl. Chem. 1953, 3, 71.

(31) Kollman, P. J . Am. Chem. Soc. 1977, 99, 4878.

(32) Erbil, H. Y. J . Adhesion Sci. Technol. 1989, 3, 29.

(33) Tamai, Y.; Matsunaga, T.; Horiuchi, K. J . Colloi I InterfaceSci. 1977, 60, 112.

(34) Lavielle, L.; Schultz, J . J . Colloid I nterfaceSci. 1985, 106, 438. medium, eq 17 becomes

$$
\gamma_{\mathrm{SH}}-\gamma_{\mathrm{SD}}=\gamma_{\mathrm{DH}} \cos \theta
$$

where subscripts $\mathrm{H}$ and $\mathrm{D}$ refer to hydrocarbon and polar (drop) liquids, respectively.

When, a hydrocarbon drop is placed in the pol ar liquid medium, eq 17 becomes

$$
\gamma_{\mathrm{SB}}-\gamma_{\mathrm{SH}}=\gamma_{\mathrm{HB}} \cos \theta
$$

where subscript B refer to polar liquid (bulk) media. By combining eqs $1,10 \mathrm{a}$, and $13 \mathrm{a}$, one obtains theinterfacial tension expression

$$
\begin{aligned}
\gamma_{12}=\left\{\left(\gamma_{1}{ }^{\mathrm{LW}}\right)^{1 / 2}-\left(\gamma_{2}{ }^{\mathrm{LW}}\right)^{1 / 2}\right\}^{2}+2\left\{\left(\gamma_{1}^{+}\right)^{1 / 2}-\right. \\
\left.\left(\gamma_{2}^{+}\right)^{1 / 2}\right\}\left\{\left(\gamma_{1}^{-}\right)^{1 / 2}-\left(\gamma_{2}^{-}\right)^{1 / 2}\right\}
\end{aligned}
$$

By combining eqs 18 and 20 and by taking $\gamma_{\mathrm{H}}=\gamma_{\mathrm{H}} \mathrm{LW}$ and $\gamma_{H^{+}}=\gamma_{H^{-}}=0$, since hydrocarbons areapolar, one obtains

$$
\begin{aligned}
\gamma_{H}-\gamma_{D}+ & 2\left\{\left(\gamma_{S}{ }^{L W} \gamma_{D}{ }^{L W}\right)^{1 / 2}-\left(\gamma_{S}{ }^{L W} \gamma_{H}\right)^{1 / 2}+\right. \\
& \left.\left(\gamma_{S}^{+} \gamma_{D}^{-}\right)^{1 / 2}+\left(\gamma_{D}^{+} \gamma_{S}^{-}\right)^{1 / 2}\right\}=\gamma_{D H} \cos \theta
\end{aligned}
$$

Similarly, by combining eqs 19 and 20, one obtains

$$
\begin{aligned}
\gamma_{B}-\gamma_{H}+ & 2\left\{\left(\gamma_{S}{ }^{L W} \gamma_{H}\right)^{1 / 2}-\left(\gamma_{S}{ }^{L W} \gamma_{B}{ }^{L W}\right)^{1 / 2}-\right. \\
& \left.\left(\gamma_{S}^{+} \gamma_{B}^{-}\right)^{1 / 2}-\left(\gamma_{S}^{-} \gamma_{B}^{+}\right)^{1 / 2}\right\}=\gamma_{H B} \cos \theta
\end{aligned}
$$

Equations 21 and 22 are used to calculate $\gamma_{\mathrm{S}}{ }^{\mathrm{LW}}, \gamma_{\mathrm{S}}{ }^{+}$, and $\gamma_{s}{ }^{-}$values by using at least three liquid drops in the immiscible medium.

Although the two-liquid method is theoretically acceptable, in practice a difficulty exists with thetwo-liquid method: In the one-liquid method the advancing contact angle is measured because that is the only contact angle by which thetriple point (gas/liquid/solid) is such that the liquid is in contact with a nonpreviously wetted solid surface. With the two-liquid method the advancing contact angle made by the first liquid is, ipso facto, the retreating contact angle by the second liquid. The retreating anglecan only yield flawed information, because it shows the hybrid interaction energy between a given liquid and a solid surface prewetted by that liquid. Thus, in practice the polymer surface is al ways soiled by one of theliquids. However the two-liquid method was found to besuitabl eto investigatetherestructuring of the polymer chains in different environments. ${ }^{13-16,25,33}$

Critical SurfaceTension. An empirical organization of contact angle data on polymers was introduced by Zisman and co-workers. ${ }^{35,36}$ They plotted $\cos \theta$ versus $\gamma_{L}$ of the drop liquids (one-liquid method) and even for a variety of nonhomologeous liquids, the graphical points fell closetoa straight lineor collected around it in a narrow rectilinear band

$$
\cos \theta=1-\beta\left(\gamma_{\mathrm{L}}-\gamma_{\mathrm{C}}\right)
$$

Each line extrapolates to zero $\theta$ at a certain $\gamma_{\mathrm{L}}$ value, which Zisman has called the "critical surface tension of solid", $\gamma_{\mathrm{c}}$. Zisman warned that $\gamma_{\mathrm{c}} \neq \gamma_{\mathrm{SV}}$, and $\gamma_{\mathrm{c}}$ is an empirical value, characteristic of a given solid; however, $\gamma_{\mathrm{Sv}}$ is a thermodynamic quantity. ${ }^{36} \beta$ is a coefficient which is approximately $0.03-0.04$.

(35) Fox, H. W.; Zisman, W. A. J . Coll oid I nterfaceSci. 1952, 7, 428. (36) Zisman, W. A. In Contact Angle, Wettability and Adhesi on; Adv. Chem. Ser. No. 43; American Chemical Society: Washington D.C., 1964; p 1. 


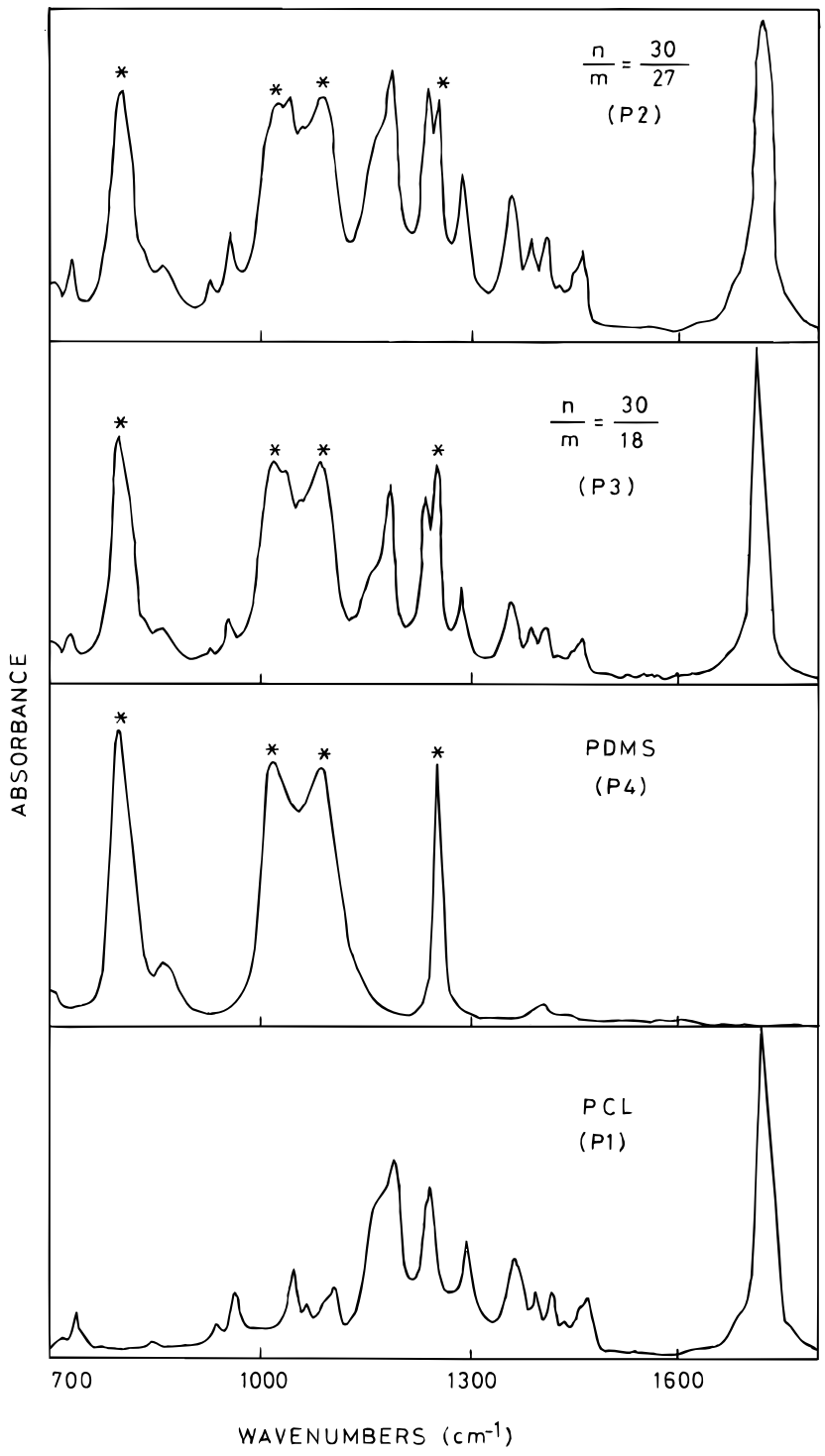

Figure 1. FTIR absorbancespectra of purepoly( $\epsilon$-caprolactone) (P1), pure poly(dimethylsiloxane) (P4), PCL/PDMS/PCL copolymers with siloxane to caprolactone ratio 30/18 (P3) and 30/27 (P2).

The $\gamma_{c}$ concept was found to be useful in classifying polymer surfaces. However, there are objections to this method; the value of $\gamma_{c}$ is often uncertain since the extrapolation is quite long and $\gamma_{c}$ is assumed to be independent of the nature of the drop liquid, but the reverse is true especially for acid-base interacting liquids. ${ }^{20}$ It was proposed ${ }^{21}$ that this method is only valid for measurements with completely apolar liquids, yiel ding only $\gamma_{\mathrm{S}}{ }^{\mathrm{LW}}$, and can be used if $\cos \theta$ is plotted Vs $\left(\gamma_{\mathrm{L}}^{\mathrm{LW}}\right)^{1 / 2}$ and not vs $\gamma_{L}{ }^{\mathrm{LW}}$ or $\gamma_{L}{ }^{37}$

\section{Results and Discussion}

FTIR. Figure 1 depicts the FTIR spectra (in the absorbance mode) of the pure PCL and PDMS as well as the P2 and P 3 copolymer films. Thestrong carbonyl peak at $1700 \mathrm{~cm}^{-1}$ can be used to qualitatively estimate the bulk composition of PCL and the strong peaks at 720 and $1025 \mathrm{~cm}^{-1}$ can be used for PDMS. The FTIR estimated bulk compositions are given in Table 2 . The $\mathrm{Si}-\mathrm{O} / \mathrm{C}=\mathrm{O}$ ratio was found to be 0.58 for the $\mathrm{P} 2$ sample and 0.67 for the P3 sample. When compared with the mole percent of PDMS in Table1, theenhancement of PDMS at thesurface

(37) Good, R. J . J . Colloid Interface Sci. 1977, 59, 398. was qualitatively shown by FTIR. However, the depth of penetration of the FTIR technology is about 400-2000 $\mathrm{nm}$, which is many thousands of molecular layers, and this deeper penetration suggests that FTIR technol ogy is not as sensitive as ESCA in discriminating between the surface chemistry changes at the upper layers. ${ }^{12,38,39}$

ESCA. This method involves the irradiation of solid materials in vacuo with monoenergetic soft X-rays and sorting the emitted el ectrons by energy. The spectrum obtained is a plot of the number of emitted electrons vs kinetic energy. Since the mean free path of the electron is small, the electrons that are detected originate from only the top few layers. ${ }^{40}$ ESCA offers the possibility of obtaining quantitativeinformation on compositions in not only the immediate surface but also the subsurface typically to a depth of 3-10 nm. .,10 $^{8}$

In order to calibrate the atomic sensitivity factor ratio of carbon 1stosilicon 2p and toidentify thecharacteristics on the spectrum, ESCA spectra of PDMS and PCL homopolymers weretaken and are shown in F igure 2 with spectra of $\mathrm{P} 2$ and $\mathrm{P} 3$ copolymers. PCL gives two peaks in the $\mathrm{C}$ is region. Themain peak at lower binding energy corresponds to the saturated $\mathrm{C}$ atoms and the smaller peak at higher binding energy arises from theunsaturated carbons. However, PDMS gives only one $C$ ls peak showing that all thecarbon atoms in PDMS aresaturated and bonded in the same way.

A careful examination of Figure 2 shows that the Si $2 p$ peaks (as well as Si KKL Auger peaks which arenot shown here) are fairly narrow (full width at half maximum) < $1.1 \mathrm{eV}$ ) and can be assigned to only one chemical state, whereas the $C$ is peaks are broad and consist of multiple states (except in pure PDMS) and can be curve-fitted to three peaks assigned to hydrocarbon $(\mathrm{CH})$, etheric $(\mathrm{C}-$ $\mathrm{O})$, and esteric $(\mathrm{O}-\mathrm{C}=\mathrm{O})$ type of moieties at around 285.0, 286.5, and $289.1 \mathrm{eV}$, respectively. The esteric peak is well resolved in all cases and is indicative of poly $(\epsilon-$ caprolactone). Therefore, for quantitative evaluation of surface composition of the copolymers, either the Si 2p/C 1s (total) or the Si 2p/C 1s (C=O) peak ratios can be used. $\mathrm{Si} / \mathrm{C}$ (total) atomicratios areobtained fromSi $2 \mathrm{p} / \mathrm{C}$ 1s ratio (after correction for the cross section and energy dependence) and aregiven in Table2. In this paper, thesurface compositions of PCL/PDMS/PCL triblock copolymers were calculated from Si/C (total) atomicratios as usual. ${ }^{10}$ Since, the $C$ 1s peak area ratios for $90^{\circ}$ and $20^{\circ}$ takeoff angles are identical and Si 2 p peak ratios are very close to each other, only the data of $20^{\circ}$ takeoff angle is used in surface composition calculations. Thesurfacecompositions of the samples by ESCA measurements are given in Table 3 . It is recognized that in general, the surface concentration of PCL segments decreases with the decrease of the length of that segment in the bulk. P2 copolymer has a molar concentration of $29.8 \%$ at the surface having $2 \times 27=54$ units of PCL with 31 units of PDMS in bulk, whereas P3 copolymer has a molar concentration of $20.8 \%$ at the surface having $2 \times 18=36$ units of PCL with the same 31 units of PDMS in bulk. Conversely, it can besaid that the surface concentration of PDMS increases with the increase of therelativeconcentration of PDMS in thebulk.

Themolar concentration of PDMS increased from 36.5\% in the bulk up to $70.2 \%$ in the surfacefor sampleP2, which is equivalent to a $92 \%$ enrichment of the low surface tensi on PDMS segments at the surface. For sample P3, PDMS molar concentration increased from $46.3 \%$ in the

(38) Filbey, J . A.; Wightman, J . P. In Adhesive Bonding; Lee, L. H., Ed.; Plenum Press: New York, 1991; p 175.

(39) Giroux, T. A.; Cooper, S. L. J. Appl. Polym. Sci. 1991, 43, 145. (40) Mullenberg, G. E., Ed. Handbook of Photoel ectron Spectroscopy; Perkin Elmer, Eden Praire, MN, 1979. 
Table 2. ESCA and FTIR Measurements of PDMS, PCL, and Their Copolymers

\begin{tabular}{|c|c|c|c|c|c|c|c|c|c|c|c|c|c|c|}
\hline & & \multicolumn{8}{|c|}{ XPS } & \multicolumn{5}{|c|}{ IR } \\
\hline & & \multirow[b]{2}{*}{ O ls } & \multicolumn{3}{|c|}{ C 1s } & \multirow[b]{2}{*}{ Si $2 p$} & \multirow[b]{2}{*}{$S i_{K L L}{ }^{a}$} & \multirow[b]{2}{*}{ Si/C (total) } & \multirow[b]{2}{*}{$\mathrm{Si} / \mathrm{C}=\mathrm{O}$} & \multicolumn{2}{|c|}{$\mathrm{Si}-\mathrm{O}$} & \multicolumn{2}{|c|}{$\mathrm{C}=\mathrm{O}$} & \multirow[b]{2}{*}{$\mathrm{Si}-\mathrm{O} / \mathrm{C}=\mathrm{O}$} \\
\hline & & & $\mathrm{CH}$ & $\mathrm{C}-\mathrm{O}$ & $\mathrm{C}=\mathrm{O}$ & & & & & $\mathrm{v}$ & Abs. & $\mathrm{v}$ & Abs. & \\
\hline $\begin{array}{l}\mathrm{PCL} \\
(\mathrm{P} 1)\end{array}$ & $\begin{array}{l}\text { B.E. (eV) } \\
\text { intensity at } 90^{\circ} \\
\text { at } 20^{\circ}\end{array}$ & $\begin{array}{r}532.7 \\
(0.43) \\
(0.50)\end{array}$ & $\begin{array}{c}285.0 \\
(0.64) \\
(0.64)\end{array}$ & $\begin{array}{r}286.5 \\
(0.21) \\
(0.21)\end{array}$ & $\begin{array}{r}289.0 \\
(0.15) \\
(0.15)\end{array}$ & & & & & & & 1725 & 2.00 & \\
\hline $\begin{array}{l}(30 / 27) \\
(\mathrm{P} 2)\end{array}$ & $\begin{array}{l}\text { B.E. (eV) } \\
\text { intensity at } 90^{\circ} \\
\text { at } 20^{\circ}\end{array}$ & $\begin{array}{c}532.5 \\
(0.58) \\
(0.60)\end{array}$ & $\begin{array}{c}285.0 \\
(0.76) \\
(0.76)\end{array}$ & $\begin{array}{c}286.0 \\
(0.16) \\
(0.16)\end{array}$ & $\begin{array}{c}289.1 \\
(0.08) \\
(0.08)\end{array}$ & $\begin{array}{c}102.2 \\
(0.27) \\
(0.26)\end{array}$ & 1607.8 & 0.22 & 2.8 & 1027 & 0.45 & 1726 & 0.77 & 0.58 \\
\hline $\begin{array}{l}\text { (30/18) } \\
\text { (P3) }\end{array}$ & $\begin{array}{l}\text { B.E. (eV) } \\
\text { intensity at } 90^{\circ} \\
\text { at } 20^{\circ}\end{array}$ & $\begin{array}{r}532.4 \\
(0.65) \\
(0.70)\end{array}$ & $\begin{array}{c}285.0 \\
(0.77) \\
(0.77)\end{array}$ & $\begin{array}{r}286.5 \\
(0.18) \\
(0.18)\end{array}$ & $\begin{array}{c}289.2 \\
(0.05) \\
(0.05)\end{array}$ & $\begin{array}{c}102.2 \\
(0.34) \\
(0.33)\end{array}$ & 1607.7 & 0.28 & 5.2 & 1027 & 0.62 & 1726 & 0.92 & 0.67 \\
\hline $\begin{array}{l}\text { PDMS } \\
\text { (P4) }\end{array}$ & $\begin{array}{l}\text { B.E. (eV) } \\
\text { intensity at } 90^{\circ} \\
\text { at } 20^{\circ}\end{array}$ & $\begin{array}{r}532.0 \\
(0.78) \\
(0.85)\end{array}$ & $\begin{array}{r}284.6 \\
(1.00) \\
(1.00)\end{array}$ & & & $\begin{array}{r}102.1 \\
(0.62) \\
(0.59)\end{array}$ & 1607.8 & 0.50 & & 1025 & 2.00 & & & \\
\hline
\end{tabular}

a KLL Auger energy in eV.

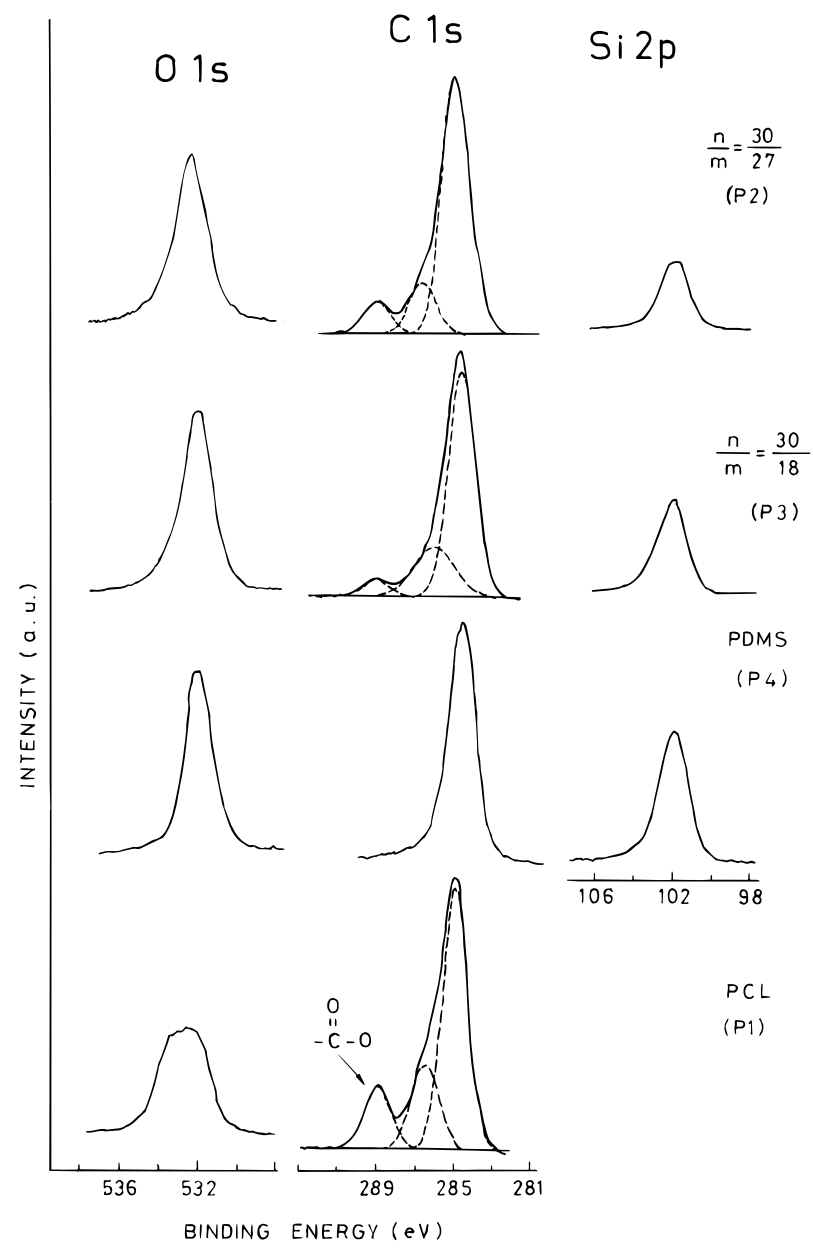

Figure 2. $O 1 s, C 1 s$, and $S i 2 p$ regions of ESCA spectra recorded at $90^{\circ}$ takeoff angle and using $\mathrm{Mg} \mathrm{K} \alpha \mathrm{X}$-rays of polymers as in Figure 1.

Table 3. Surface Composition of Samples by ESCA Measurements

\begin{tabular}{cccc}
\hline sample & $\%$ mol PDMS & $\%$ mol PCL & $\mathrm{C}=\mathrm{O} / \mathrm{C}$ 1s (total) \\
\hline P1 & & 100.0 & 0.15 \\
P2 & 70.2 & 29.8 & 0.08 \\
P3 & 79.2 & 20.8 & 0.05 \\
P4 & 100 & &
\end{tabular}

bulk up to $79.2 \%$ in the surface, which is equivalent to a $71 \%$ enrichment of PDMS segments at the surface.

Since values of cohesi ve energy of the two blocks are quite different $\left(\delta=15.3 \mathrm{MPa}^{1 / 2}\right.$ for PDMS and $\delta=19.2$ $\mathrm{MPa}^{1 / 2}$ for $\left.\mathrm{PCL}^{41}\right)$, a microphase-separated morphology of this copolymer is expected where the PDMS spherical microdomains are segregated in the surface region to minimize the surface energy of the copolymer. It was reported that ${ }^{5}$ despite the amorphous PDMS block, P2 and $\mathrm{P} 3$ copolymers are semicrystalline and X-ray diffractometry studies lead a microphase separation of amorphous, PDMS-rich regions versus crystalline, PCL-rich ones. The P3 copolymer melts at $54{ }^{\circ} \mathrm{C}$ and the P2 copolymer, having longer PCL blocks than P3, melts at $57^{\circ} \mathrm{C}$, only slightly lower than pure PCL. ${ }^{5}$ There is a competition between phase separation and PCL crystallization tocontrol thesurfacecomposition and morphology of P2 and P 3 copolymers. Since P 2 copolymer has longer PCL blocks and higher crystallinity, thetendency of phase separation is higher for this copolymer than for the P3 copolymer, which results in 92\% PDMS enrichment for P2, which is higher than the 71\% PDMS enrichment for P3.

As seen from Table 3, the highest PDMS molar percent at the surface is $79.2 \%$ and it is not $100 \%$ as reported previously for some PDMS copolymers. ${ }^{9}$ This can be explained by the molecular weight of the PDMS segment being approximately 2300 in both copolymers, a valuefar behind the reported value of 20000-25000 when the topmost surface region of the copolymer is composed of nearly $100 \%$ PDMS. $^{9}$ Furthermore, $72.5-84.9 \%$ PDMS at surface concentration was reported for PDMS- ureaurethane segmented copolymers having PDMS segment molecular weight of 2400, which is very similar to our results. ${ }^{9}$

One feature of PCL/PDMS/PCL copolymers is that the PDMS surface composition does not change substantially when the ESCA sampling depth varies from 4 to $10 \mathrm{~nm}$ (for 20-90 takeoff angle), due to the semicrystalline nature of the copolymer. However, it was reported that for some PDMS block copolymers, the amount of PDMS attenuates as the ESCA sampling increases. ${ }^{10,42}$

As mentioned earlier, another indication of the decrease of PCL blocks at thesurfaceis the decrease of $C$ is $(C=O) / C$ 1s esteric peak ratios. The stoichiometric ratio of pure PCL is 0.167 from (1:5) molar ratio of pure PCL. ESCA analysis showed a figure of 0.15 as shown in Table 3 , indicating that the structuring of pure PCL under high vacuum does not favor $\mathrm{C}=\mathrm{O}$ surface enrichment. As seen in Table 3, this ratio decreases to 0.08 for $\mathrm{P} 2$ and 0.05 for $\mathrm{P3}$, indicating the decrease of PCL segment (or theincrease of PDMS segments) at the surface. Theseresults support theexperimental findingsusing theSi $2 \mathrm{p} / \mathrm{C}$ 1s (total) ratios which are given in Table 1.

(41) Hubbell, D. S.; Cooper, S. L.J . Appl. Polym. Sci. 1977, 21, 3035. 4601.

(42) Chen, X.; Lee, H. F.; Gardella, J. A. Macromolecules 1993, 26 
Table 4. Surface Tension Components of the Testing Liquids at $20^{\circ} \mathrm{C}$ in $\mathrm{mJ} / \mathrm{m}^{2} 20$

\begin{tabular}{lllccc}
\hline & $\gamma \mathrm{L}$ & $\gamma_{\mathrm{L}}{ }^{\mathrm{LW}}$ & $\gamma_{\mathrm{L}}{ }^{\mathrm{AB}}$ & $\gamma_{\mathrm{L}^{+}}$ & $\gamma_{\mathrm{L}^{-}}$ \\
\hline water & 72.8 & 21.8 & 51 & 25.50 & 25.5 \\
glycerol & 64 & 34 & 30 & 3.92 & 57.4 \\
formamide & 58 & 39 & 19 & 2.28 & 39.6 \\
ethylene glycol & 48 & 29 & 19 & 1.92 & 47.0 \\
paraffin oil & 28.9 & 28.9 & 0 & 0 & 0
\end{tabular}

Table 5. Contact Angle Values (in degrees) on Sample Polymers $^{43}$

\begin{tabular}{ccccccc}
\hline sample & water & glycerol & formamide & $\begin{array}{c}\text { ethylene } \\
\text { glycol }\end{array}$ & $\begin{array}{c}\text { diethylene } \\
\text { glycol }\end{array}$ & $\begin{array}{c}\text { paraffin } \\
\text { oil }\end{array}$ \\
\hline P1 & 89 & 72 & 63 & 68 & 54 & 33 \\
P2 & 93 & 86 & 81 & 77 & 71 & 36 \\
P3 & 92 & 73 & 69 & 64 & 68 & 30
\end{tabular}

Critical SurfaceTension of Polymers. Thesurface tension values of the test liquids used in contact angle determinations are given in Table $4 .^{20}$ The measured contact anglevalues of polymericsamples in theone-liquid method are given in Table 5.43 A $\cos \theta$ versus $\gamma_{\mathrm{L}}$ pl ot is given in Figure 3 . Linear regression is applied to extrapolate each linetozero $\theta$ in order to cal culatecritical surface tension values of polymer samples. $\gamma_{c}$ values of 19,14 , and $18 \mathrm{~mJ} / \mathrm{m}^{2}$ are found for P1, P2, and P3 samples, respectively. As seen in Figure 3, only poor results can be obtained by this method when polar and apolar liquid drops are used together. F or homopolymer PCL (P1), this value is too low. For P2 and P 3 the magnitudes of $\gamma_{c}$ are reasonable because of the siloxane enrichment at the surface. However, $\gamma_{c}$ of P2 $>\gamma_{c}$ of P3 was expected from ESCA measurements, but the reverse result is obtained. Since the extrapolations are quite long, the values of $\gamma_{c}$ seem to be uncertain. (When a $\cos \theta$ vs $\left(\gamma_{\mathrm{L}}{ }^{\mathrm{LW}}\right)^{1 / 2}$ graph is plotted, no improvement was seen). In addition, for pure PCL $\gamma_{c}$ does not reflect its acid-base interacting properties. Consequently, it can be stated that this method is unsatisfactory and gives only a rough estimate of theorder of the solid surface tensions.

One-Liquid Method Evaluation. Contact angle measurements can in appropriate instances provide information on the gross structure of theimmediateouter boundary of a polymer. Contact angles relate to the fractions of nonpolar and acid-baseinteractingstructural elements on a surface. Comparison of the water contact angl es of the polymer samples can give an idea about the hydrophilicity of the surfaces. It is known that PDMS exhibits high hydrophocibity ${ }^{3,44}$ and gives water contact angles higher than $90^{\circ}$. The high water contact angles indicate the presence of a more complete overlayer of PDMS at the copolymer surface. ${ }^{11}$ In Table5, an increase of water contact angle was seen for P2 and P3 samples over P1, parallel with the increase in PDMS content. However, there is only $3-4^{\circ}$ difference between sample $\mathrm{P} 1$ and samples $\mathrm{P} 2$ and $\mathrm{P} 3$ and only $1^{\circ}$ difference between P2 and P3 samples, indicating two points: First, water contact angles alone are only a rough guide to evaluate hydrophilicity, and second, poly( $\epsilon$-caprolactone) is not so hydrophilic at the surface as expected. Then water, glycerol, formamide, ethylene glycol, and paraffin oil contact angle values were used to calculate $\gamma_{\mathrm{s}}{ }^{\mathrm{LW}}, \gamma_{\mathrm{s}}{ }^{\mathrm{AB}}$, $\gamma_{S}{ }^{+}, \gamma_{S}{ }^{-}$, and $\gamma_{S}{ }^{\text {TOT }}$ values of the three polymer samples by using eq 16. First, $\gamma_{\mathrm{s}}{ }^{\mathrm{LW}}$ values were calculated using apolar paraffin oil contact angle results and are reported in Table 6. It was seen that this value ranges between 23.6 and $25.2 \mathrm{~mJ} / \mathrm{m}^{2}$ with a $3 \%$ deviation from the mean

(43) Yaşar, B. Msc. Thesis, Boğaziçi University, I stanbul, 1996. 2039.

(44) Inone, H.; Ueda, A.; Nagai, S. J. Appl. Polym. Sci. 1988, 35,

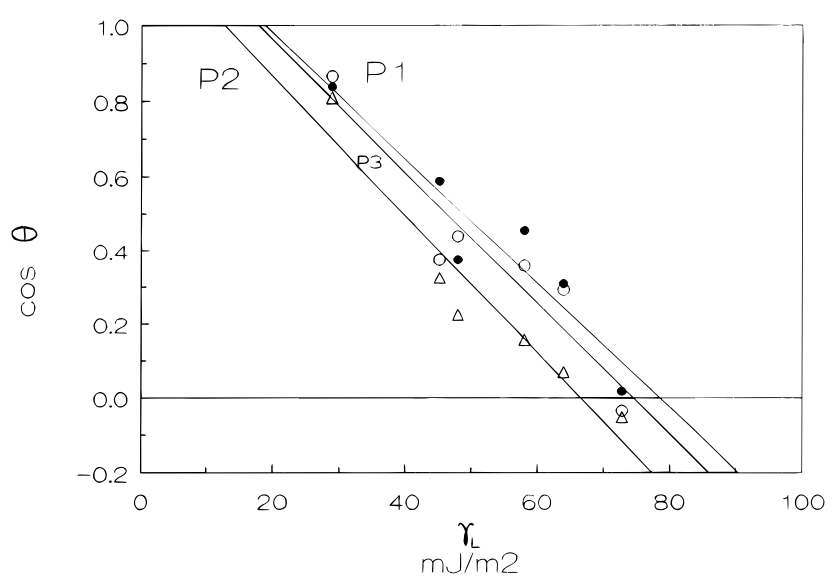

Figure 3. Critical surfacetensions of $\mathrm{P} 1, \mathrm{P} 2$, and $\mathrm{P} 3$ polymers plotted using contact angle data.

Table 6. One-Liquid Contact Angle Method Results at 20 ${ }^{\circ} \mathrm{C}$ in $\mathrm{mJ} / \mathrm{m}^{2} 43$

\begin{tabular}{cccccc}
\hline sample & $\gamma_{\mathrm{S}}{ }^{\mathrm{LW}}$ & $\gamma_{\mathrm{S}^{+}}$ & $\gamma_{\mathrm{S}^{-}}$ & $\gamma_{\mathrm{S}}{ }^{\mathrm{AB}}$ & $\gamma_{\mathrm{S}}{ }^{\text {TOT }}$ \\
\hline P1 & 24.4 & 0.22 & 5.24 & 2.1 & 26.5 \\
P2 & 23.6 & 0.05 & 4.47 & 0.9 & 24.5 \\
P3 & 25.2 & 0.61 & 2.35 & 2.4 & 27.6
\end{tabular}

and may be accepted as constant for all three samples. Subsequently, by using the known $\gamma_{s}{ }^{\mathrm{LW}}$ values eq 16 was solved for two unknowns using water-formamide, waterethylene glycol, and water-glycerol pairs, and only minimum $\gamma_{s}{ }^{+}$and maximum $\gamma_{s}{ }^{-}$values are reported in Table6. (Since, $\gamma_{\mathrm{s}}{ }^{+}$is an electron acceptor (or Lewis acid) parameter of polymer surface tension and poly( $\epsilon$-caprolactone) having (-COO) groups) at the surface is a hydrophilic basic polymer, $\gamma_{\mathrm{s}}{ }^{+}$should beclosetozero, this is the reason for reporting only minimum $\gamma_{s}{ }^{+}$and maximum $\gamma_{s}{ }^{-}$values.) As seen from Table 6 , there is a decreasing basicity $\left(\gamma_{S^{-}}{ }^{-}\right)$with the increase of PDMS content on the surface of the copolymers. Theresults are in agreement with the ESCA results shown in Table 3; however, they are not proportional to them because $\mathrm{PCL}$ mole percent decreases from 100 to $30 \%$ resulting in a $\gamma_{\mathrm{s}}$ decrease from 5.24 down to only $4.47 \mathrm{~mJ} / \mathrm{m}^{2}$, but any further decrease in PCL (as mole percent) from 30 to $21 \%$, resulted in a very sharp $\gamma_{\mathrm{S}}{ }^{-}$decrease from 4.47 to 2.35 $\mathrm{mJ} / \mathrm{m}^{2}$. This can be explained for P2 copolymer; the hydrophilic PCL segments dominate at the interface in polar liquid drop mediumand show higher basic properties than expected. This situation is a typical molecular restructuring process at the copolymer surface in polar environments, where it is energetically more favorable for the polymer surface to becomeenriched in hydrophilic PLC segments and thus reducethedensity of apolar PDMS segments. The main driving force for the rearrangement of the molecules at the surface is the strong interaction between polar liquid and hydrophilicPCL segments. One can speculate that the PCL segments in the 3 copolymer cannot adequately restructure at the surface and cannot increase their density because of the shorter segment lengths of $\mathrm{PCL}$ in $\mathrm{P} 3$ with respect to $\mathrm{P} 2\left(\mathrm{M}_{\mathrm{w}} \sim 2000\right.$ and 3000, respectively).

Thetotal surfacetension of all the threesamples ranged between 24.5 and $27.6 \mathrm{~mJ} / \mathrm{m}^{2}$ with a $6 \%$ deviation from the mean. Although, $\gamma_{S}{ }^{\text {TOT }}$ values are affected by the $\gamma_{S}{ }^{A B}$ values and the obtained $\gamma_{\mathrm{S}}{ }^{\mathrm{AB}}$ values were not consistent; $6 \%$ deviation can beregarded negl igibleand the only main figure in Table 6 is the $55 \%$ decrease of basicity coefficient of the copolymer surface tension $\left(\gamma_{\mathrm{S}}{ }^{-}\right)$from pure $\mathrm{PCL}$ to P3 copolymer. This property is important when Tegomers (P2 and P3) are used as a polymer blend modifier, 
Table 7. Surface and Interfacial Tensions of the Testing Liquids in the Two-Liquid Method at $20^{\circ} \mathrm{C}$ in $\mathrm{mJ} / \mathrm{m}^{245}$

\begin{tabular}{lcccc}
\hline & & \multicolumn{3}{c}{$\gamma_{L_{1} L_{2}}$} \\
\cline { 3 - 5 } & $\gamma_{L}$ & water & ethylene glycol & formamide \\
\hline n-hexane & 17.91 & 50.87 & 16.28 & 25.74 \\
n-octane & 21.14 & 50.51 & 17.32 & 25.73 \\
cyclohexane & 24.38 & 49.78 & 15.46 & 24.74 \\
paraffin oil & 28.90 & 52.00 & 17.50 & 26.00
\end{tabular}

especially in polar environments, indicating that basic carboxyl groups enrich at the surface in environments containing acidic groups.

Two-Liquids Method Evaluation. The interfacial tension values between polar liquids and apolar hydrocarbons are taken from Matsunaga and I kada ${ }^{45}$ and are given in Table 7. The contact anglevalues of pol ar liquid drops on samplepolymers in hydrocarbon $(\mathrm{HC})$ media are given in Table8. SinceP3 copolymer rapidly dissol ves in most of the hydrocarbons, only paraffin oil values can be determined. (P2 copolymer was also attacked by HC solvents sl owly and it takes approximately 15 min to swell, so initial contact angle values can be succesfully measured.) The contact angle values of $\mathrm{HC}$ drops on sample polymers in polar liquid media are given in Table 9. In order to evaluate the results derived from Table 8, eq 21 is rearranged so that

$$
y=\left(\gamma_{s}^{L W}\right)^{1 / 2} x+1
$$

where

$$
\begin{gathered}
y=\gamma_{D}-\gamma_{P}+\gamma_{D P} \cos \theta \\
x=2\left\{\left(\gamma_{D}{ }^{L W}\right)^{1 / 2}-\left(\gamma_{P}\right)^{1 / 2}\right\} \\
I=2\left\{\left(\gamma_{S}^{+} \gamma_{D}^{-}\right)^{1 / 2}+\left(\gamma_{D}^{+} \gamma_{S}^{-}\right)^{1 / 2}\right\}
\end{gathered}
$$

Then $\mathrm{x}-\mathrm{y}$ plots are drawn for $\mathrm{P} 1$ and $\mathrm{P} 2$ samples and are given in Figures 4 and 5 . Linear regression was applied, and $\left(\gamma_{\mathrm{s}}^{\mathrm{LW}}\right)^{1 / 2}$ values were calculated from the slopes and I values from the intercepts of the lines in Figures 4 and 5. Values are given in Table 10. In order to evaluate the resul ts derived from Table 9 , eq 22 is rearranged identical to eq 24 , but where

$$
\begin{gathered}
\mathrm{y}=\gamma_{\mathrm{B}}-\gamma_{\mathrm{P}}+\gamma_{\mathrm{PB}} \cos \theta \\
\mathrm{x}=2\left\{\left(\gamma_{\mathrm{B}}{ }^{\mathrm{LW}}\right)^{1 / 2}-\left(\gamma_{\mathrm{P}}\right)^{1 / 2}\right\} \\
\mathrm{I}=2\left\{\left({\gamma_{\mathrm{S}}}^{+} \gamma_{\mathrm{D}}^{-}\right)^{1 / 2}+\left({\gamma_{\mathrm{D}}}^{+} \gamma_{\mathrm{S}}{ }^{-}\right)^{1 / 2}\right\}
\end{gathered}
$$

Then $\mathrm{x}-\mathrm{y}$ plots for samples $\mathrm{P} 1$ and $\mathrm{P} 2$ were constructed and are given in Figures 6 and 7 . $\left(\gamma_{\mathrm{S}}{ }^{\mathrm{LW}}\right)^{1 / 2}$ and I values which werecalculated by thelinear regression of thelines are given in Table 10 . The average $\left(\gamma_{\mathrm{S}}^{\mathrm{LW}}\right)^{1 / 2}$ for $\mathrm{P} 1$ was found to be $5.67\left(\mathrm{~mJ} / \mathrm{m}^{2}\right)^{1 / 2}$ and $2.97\left(\mathrm{~mJ}^{2} / \mathrm{m}^{2}\right)^{1 / 2}$ for P2. Since I values differ according to the drop liquid, I values for each polar liquid-polymer pair were calculated from the arithmetic mean of the intercepts of the rel evant lines in Figures 4 and 6 for $\mathrm{P} 1$ polymer and in Figures 5 and 7 for P2 polymer. Then, $\gamma_{\mathrm{s}}{ }^{+}$and $\gamma_{\mathrm{s}}{ }^{-}$values were calculated by using eq 25 (or eq 26) and their averages are given in Table 11. $\gamma_{\mathrm{s}}{ }^{\mathrm{AB}}$ values were calculated by using eq 11 and $\gamma_{s}{ }^{\text {TOT }}$ values by using eq 1 . Sincenox-y plot can bedrawn for $\mathrm{P} 3$ polymer due to its rapid dissolution in $\mathrm{HC}$ media, numerical analysis was applied to paraffin oil-polar liquid data (which is the only available data), and best fit val ues

(45) Matsunaga, T.; Ikada, Y. J . Colloid Interface Sci. 1988, 84, 8.

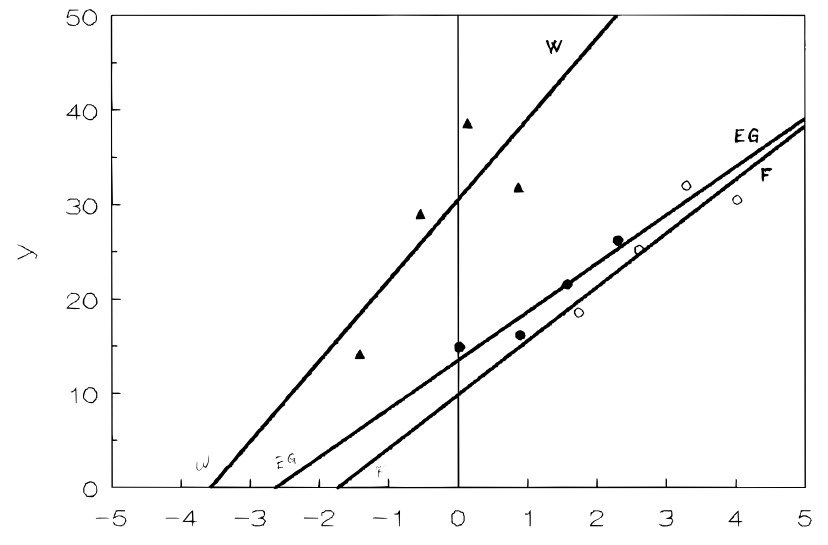

Figure 4. $\mathrm{x}-\mathrm{y}$ plot for sample $\mathrm{Pl}$ : (ム) water drops in $\mathrm{HC}$; $(\bullet$ ethylene glycol drops in $\mathrm{HC}$; (O) formamide drops in $\mathrm{HC}$.

Table 8. Contact Angle Values of the Polar Liquid Drops (in degrees) on Sample Polymers in Hydrocarbon Media in the Two-Liquid Method

\begin{tabular}{clccc}
\hline & & \multicolumn{3}{c}{ drop liquid } \\
\cline { 3 - 5 } sample & bulk liquid & water & ethylene glycol & formamide \\
\hline P1 & n-hexane & 117 & 104 & 112 \\
& n-octane & 105 & 108 & 101 \\
& cyclohexane & 113 & 119 & 110 \\
P2 & paraffin oil & 125 & 104 & 114 \\
& n-hexane & 153 & 149 & 142 \\
& n-octane & 150 & 135 & 143 \\
& cyclohexane & 145 & 138 & 149 \\
P3 & paraffin oil & 136 & 131 & 131 \\
& paraffin oil & 140 & 135 & 136
\end{tabular}

Table 9. Contact Angle Values (in degrees) of Hydrocarbon Drops on Sample Polymers in Polar Liquid Media, in the Two-Liquid Contact Angle Method

\begin{tabular}{clcccc}
\hline & & \multicolumn{4}{c}{ drop liquid } \\
\cline { 3 - 6 } sample & bulk liquid & n-hexane & n-octane & cyclohexane & $\begin{array}{c}\text { paraffin } \\
\text { oil }\end{array}$ \\
\hline P1 & water & 57 & 60 & 66 & 51 \\
& ethylene glycol & 62 & 58 & 53 & 77 \\
& formamide & 60 & 85 & 82 & 57 \\
P2 & water & 25 & 21 & 32 & 38 \\
& ethylene glycol & 29 & 23 & 19 & 50 \\
P3 & formamide & 50 & 54 & 59 & 56 \\
& water & & & & 30 \\
& ethylene glycol & & & & 36
\end{tabular}

are given in Table 11. As seen in this table, there is an decreasing basicity $\left(\gamma_{\mathrm{S}}{ }^{-}\right)$with the increase of the PDMS content on the surface of the copolymers. However, the decrease in $\left(\gamma_{\mathrm{S}}{ }^{-}\right)$is very sharp compared with the enrichment of the PDMS segments on the surface and it is nearly inversely proportional with the quantitative ESCA results. On the other hand, the decrease of acidity $\left(\gamma_{\mathrm{s}}{ }^{+}\right)$and the Lifshitz-van der Waals component $\left(\gamma_{\mathrm{s}}^{\mathrm{LW}}\right)$ is also exhi bited in Table 11, resulting in a sharp decrease of $\left(\gamma_{\mathrm{s}}^{\mathrm{TOT}}\right)$ values. The considerable decrease in $\gamma_{\mathrm{s}}{ }^{\mathrm{LW}}$ is not normal in practice and seems to be erroneous. The minimum $\gamma_{S}{ }^{\text {TOT }}$ value reported for purePDMS was known to be $16 \mathrm{~mJ} / \mathrm{m}^{2}$ and the $\gamma_{\mathrm{s}}{ }^{\text {TOT }}$ values in Table 11 are lower than this figure, indicating that the two-liquid contact angle method results are somewhat erroneous and exaggerate the differences on the surface tension components of the polymers too much. This error may arise from the fact that the surface of the solid is prewetted by the first liquid and the contact angle measured in this method is the retreating angle of the second liquid showing the hybrid interaction energy. Therefore, the most useful comparison is the one between theoneliquid contact angle 


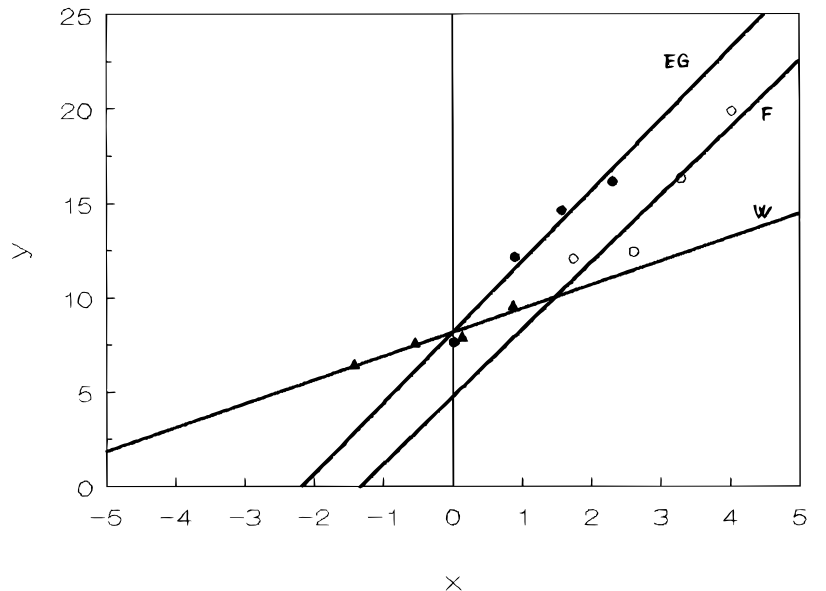

Figure 5. $x-y$ plot for sample $\mathrm{P} 2$. The symbols are the same as in Figure 4.

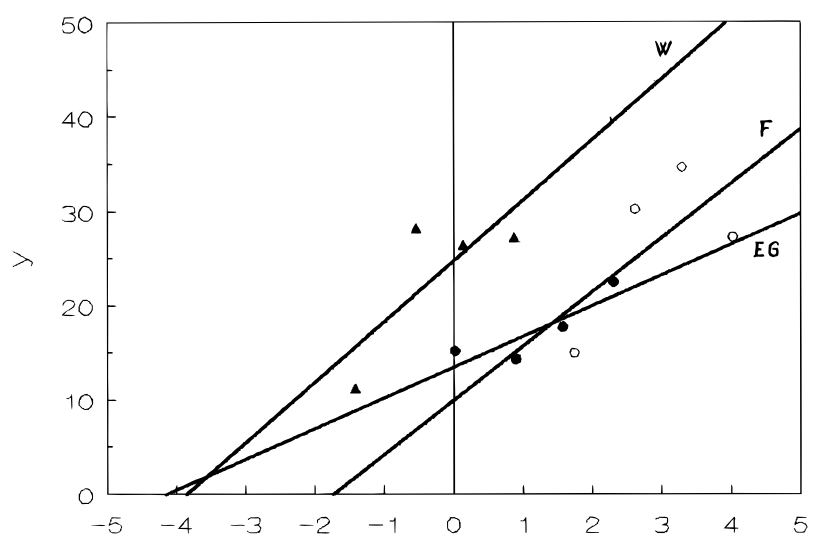

Figure 6. $\mathrm{x}-\mathrm{y}$ plot for sample $\mathrm{P1}$ : (A) HC drops in water medium; (๑) HC drops in ethyleneglycol medium; (O) HC drops in formamide medium.

Table 10. Results Obtained from the Slopes and Intercepts of the Respective Lines in Figures 4-7 in the Two-Liquid Contact Angle Method

\begin{tabular}{|c|c|c|c|c|c|}
\hline \multirow[b]{2}{*}{ medium } & \multicolumn{3}{|c|}{ P1 } & \multicolumn{2}{|c|}{ P2 } \\
\hline & drop & $\left(\gamma_{S}^{L W}\right)^{1 / 2}$ & I & $\overline{\left(\gamma_{S}{ }^{L W}\right)^{1 / 2}}$ & 1 \\
\hline $\mathrm{HC}$ & water & 8.54 & 30.35 & 1.26 & 8.20 \\
\hline $\mathrm{HC}$ & $\mathrm{EG}$ & 5.12 & 13.52 & 3.75 & 8.13 \\
\hline $\mathrm{HC}$ & $\mathrm{F}$ & 4.93 & 11.79 & 3.54 & 4.82 \\
\hline water & $\mathrm{HC}$ & 6.41 & 24.73 & 2.14 & 6.10 \\
\hline $\mathrm{EG}$ & $\mathrm{HC}$ & 3.25 & 13.50 & 3.39 & 6.84 \\
\hline $\mathrm{F}$ & $\mathrm{HC}$ & 5.76 & 9.84 & 3.75 & 9.23 \\
\hline average & & 5.67 & & 2.97 & \\
\hline
\end{tabular}

results and ESCA. However, as an approximation, when $\gamma_{\mathrm{S}}{ }^{\text {TOT }}$ values of the one and the two-liquid methods in Tables 6 and 11 are averaged, much more reasonable values for all the polymer samples are obtained which are $30.3,17.0$, and $18.0 \mathrm{~mJ} / \mathrm{m}^{2}$ for $\mathrm{P} 1, \mathrm{P} 2$, and $\mathrm{P} 3$, respectively.

The inverse proportionally of the $\gamma_{\mathrm{s}}{ }^{-}$results of the copolymers with thequantitativeE SCA results shows that the molecular restructuring at the copolymer surface in the polar environments did not take place in the twoliquid method. This may be explained by assuming that a hydrocarbon monomolecular film forms under the polar liquid drop which considerably slows down the molecular restructuring process of PCL segments.

\section{Conclusions}

1. FTIR showed the enhancement of PDMS concentration at the triblock copolymer surface with respect to the

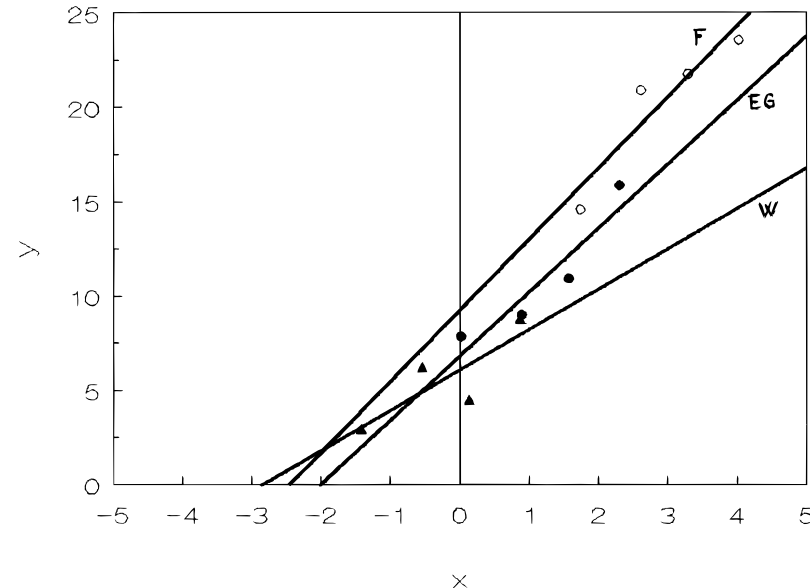

Figure 7. $x-y$ pl ot for sample $P 2$. The symbols are the same as in Figure 6.

Table 11. Two-Liquid Contact Angle Method Results

\begin{tabular}{crcccr}
\hline sample & $\gamma_{\mathrm{S}}{ }^{\mathrm{LW}}$ & $\gamma_{\mathrm{S}^{+}}$ & $\gamma_{\mathrm{S}^{-}}$ & $\gamma_{\mathrm{S}}{ }^{\mathrm{AB}}$ & \multicolumn{1}{c}{$\gamma_{\mathrm{S}}{ }^{\mathrm{TOT}}$} \\
\hline P1 & 32.1 & 0.19 & 5.30 & 2.0 & 34.1 \\
P2 & 8.8 & 0.08 & 1.06 & 0.6 & 9.4 \\
P3 & 8.2 & 0.06 & 0.29 & 0.3 & 8.5
\end{tabular}

bulk composition; however, it is a less sensitive method than ESCA and penetrates down to 400-2000 nm depth from the top of the surface, which is of limited value for many applications.

2. ESCA is a sensitive method for the determination of the surface chemical composition of the copolymers and senses a depth of 3-10 nm from the top of the surface. In our study, ESCA showed a molar concentration of PDMS of $70.2 \%$ at the surface for P2 copolymer sample which has a PDMS concentration of $36.5 \%$ in the bulk. For sample P3, PDMS concentration increased from $46.3 \%$ in the bulk up to $79.2 \%$ at the surface. ESCA results showed that in high vacuum environments, the PDMS microdomains were seggregated in the surface region to minimize the surface energy of the copolymer. Both P2 and P3 copolymers were semicrystalline, and since P2 copolymer has relatively longer PCL blocks and higher crystallinity, the tendency of phase separation is higher for this copolymer than that for the P3 copolymer. This explains the $92 \%$ enrichment for $\mathrm{P} 2$ at the surface with respect to the bulk composition, which is higher than the 71\% PDMS enrichment for P3. In other words, Ionger PCL segments favor the surface enrichment of PDMS segments. The $M_{w}$ values of PDMS segments were low (only approximately 2300) in both P2 and P 3 copolymers, and the $100 \%$ coverage of PDMS at the surface was not found unlike for some other PDMS copolymers having their PDMS segments with $M_{w} \sim 22500$, which were previously reported in the literature. Another unlike feature was that the PDMS surface composition did not changesubstantially with thechange of theE SCA takeoff angle, thus the sampling depth, duetothesemicrystalline nature of the copolymer used.

3. Contact angle measurements provide information on thefractions of thenonpolar and acid-base interacting structural elements of the outer boundary of a copolymer, which can adopt different surface compositions in different polar liquid environments (contacting liquid drops). Critical surface tension approach is an insensitive old approach and could only give a qualitativeestimate of the order of the surface tension of the copolymer samples. It did not provide information on acid-base interacting properties of all the samples. The water contact angles alone were only a rough guide to evaluate the hydrophilicity of the sample surfaces. 
4. One-liquid contact angle results were evaluated by using van Oss, Good, and Chaudhury's LW and $A B$ methodology and it was determined that the apolar LW surfacetensi on coefficients, $\gamma_{\mathrm{S}}{ }^{\mathrm{LW}}$, for all the samples were nearly constant; however, the basicity surface tension coefficients, $\gamma_{\mathrm{s}}{ }^{-}$, decreased with theincrease of the PDMS content on the surface, a result which is in agreement with theESCA results but not proportional tothem. This result indicates that a molecular restructuring process took place under the contact area of polar liquid drops, where the hydrophilic PCL segments were enriched at the surface by the molecular rearrangements and thus reduced the density of apolar PDMS segments, which were previously enriched at the surface. The driving force for this rearrangement is thestrong $A B$ interaction between the basic carboxyl groups of PCL segments and the Lewis acidicgroups of the contacting polar liquids. Onceagain, the shorter PCL segments prevent this rearrangement process to a limited extend so that the P3 copolymer containing short PCL segments cannot increaseits density at the surface as much as the $\mathrm{P} 2$ copolymer having longer PCL segments.

5. Two-liquid contact angle results were evaluated by combining the classical graphical method with van Oss, Good, and Chaudhury's approach. It was determined that the basicity surface tension coefficients, $\gamma_{\mathrm{S}}{ }^{-}$, decreased with the increase of the PDMS content on the surface, and this decrease is inversely proportional with the quantitative ESCA results. In addition, the molecular restructuring of the $P C L$ segments did not take place in the two-liquids method. However, the $\gamma_{\mathrm{s}}{ }^{\mathrm{LW}}$ coefficient for P2 and P 3 was found to decrease unreasonably, resulting in too low $\gamma_{S}{ }^{\text {TOT }}$ values, which seems to be a defect of this method probably due to the reason that the surface of the solid is prewetted by the first liquid and the contact angle measured in this method is the retreating angle of the second liquid showing the hybrid interaction energy. Therefore, the most reasonable comparison was found to bebetween theone-liquid contact angleresults and ESCA. 